Memorandum No. 1736

Optimal picking of large orders

in carousel systems

N. LITVAK

October, 2004

ISSN 0169-2690 


\title{
Optimal Picking of Large Orders in Carousel Systems
}

\author{
Nelly Litvak \\ University of Twente \\ Dep. Appl. Math., Faculty of EEMCS \\ P.O. Box 217, 7500 AE Enschede \\ The Netherlands \\ n.litvak@math.utwente.nl
}

\begin{abstract}
A carousel is an automated storage and retrieval system which consists of a circular disk with a large number of shelves and drawers along its circumference. The disk can rotate either direction past a picker who has a list of items that have to be collected from $n$ different drawers. In this paper, we assume that locations of the $n$ items are independent and have a continous non-uniform distribution over the carousel circumference. For this model, we determine a limiting behavior of the shortest rotation time needed to collect one large order. In particular, our limiting result indicates that if an order is large, then it is optimal to allocate less frequently asked items close to the picker's starting position. This is in contrast with picking of small orders where the optimal allocation rule is clearly the opposite. We also discuss travel times and allocation issues for optimal picking of sequential orders.
\end{abstract}

Keywords: carousel systems, travel times, spacings, weak convergence, allocation policy. AMS Subject classification: 90B06, 90B80, 62E15, 60F05, 60J20.

\section{Introduction}

A carousel is an automated storage and retrieval system which is widely used in modern warehouses as one of major technologies for small parts' storage. The system consists of a circular disk with a large number of shelves and drawers along its circumference. The disk rotates either direction bringing the items to a picker who has a stationary position in front of the carousel. This takes away the walking time and thus enhances efficiency of the picker. Other important benefits include a better control over materials and a greater utilization of available space.

A natural model of a carousel is a circle of length 1. An order consists of $n$ items whose locations are modeled as points on a circumference. The time needed to fulfill an order consists of a pick time (collecting the items from drawers), plus the travel (rotation) 
time of the carousel. For a given carousel unit with a human picker, the pick times are essentially pre-defined and not subject to control. The travel time however can be optimized by adopting an efficient allocation policy and choosing a picking sequence that provides a reasonably short route along the circle. Thus, one of relevant research problems in carousel systems is to characterize the travel time for all kinds of lay-outs and various picking sequences.

It was noticed by many authors (see e.g. Bartholdi and Platzman [2]) that the shortest possible route has at most one turn. Such route can be also successfully approximated by socalled $m$-step strategies proposed in [14] and analyzed in detail in [9]. These strategies allow at most one turn where the number of items collected before the turn can not exceed some fixed number $m \geq 0$. In the recent paper of Meller and Klote [11], a no-reversal strategy is adopted. This is in fact an $m$-step strategy with $m=0$, i.e., the carousel rotates either only clockwise or only counterclockwise choosing the shortest of the two possible routes. In practice, the nearest item heuristic is often applied. Under this heuristic, the next item to be collected is always the nearest one.

The analysis of the travel time under various strategies is in general a non-trivial problem. This problem however has been resolved for independent uniformly distributed items' locations. References $[7,8]$ provide a complete analysis of the nearest item heuristic including the distribution of the travel time, the moments, the asymptotic behavior when $n$ goes to infinity and the distribution for the number of turns. The analysis leads to surprising results such as strikingly simple distribution of the travel time and the independence of the travel time and the number of turns! In [9], this kind of results were obtained for the $m$-step strategies. The shortest route has been analyzed in [10].

The results on the order picking time in one carousel can be further applied in performance analysis for more complex warehousing systems. For instance, in [11] the expected travel time in one carousel is needed in order to evaluate the throughput for a carousel pod. An interesting analysis of two carousels operated by one picker was presented by Park et al. [12] and considerably extended by Vlasiou et al. [15, 16]. For such analysis, the full knowledge of the travel time distribution is required.

In this paper, we focus on the length of the shortest rotation time needed to collect one order when the order size $n$ is large and the items' locations have a non-uniform continuous distribution with a positive density $f$ on $[0,1]$. This model reflects a relevant situation when some of the drawers are required more frequently than others. One peculiar case of nonuniform items' locations was studied by Wan and Wolff [17] who analyzed the problem of picking clumpy orders, i.e. the orders concentrated on a relatively small segment of a circle. To the best of our knowledge, there is no other paper dealing with travel times in carousel systems with non-uniform items' locations. In the present work, we completely characterize the limiting behavior of the travel time when the number of items goes to infinity. The limiting behavior is similar to the one derived in [10] for the uniform case but the formula involves the values of $f$ near the picker's starting point. Also, we analyze the limiting distribution of the number of items collected before a turn. In the uniform case, this distribution is known to be geometric with parameter $1 / 2$ (see $[9,10])$. In this paper, we show that this surprising result also holds for non-uniform items' locations. 
In a system where different items are demanded with distinct frequencies, it is important not only to choose a short route but also to allocate the items on a carousel in such a way that the travel time is reduced. If an order consists of one item, then so-called organpipe allocation is proved to be optimal [3]. However, the allocation problem has not yet been resolved neither for an order size greater than one, nor for multiple carousels. The latter problem was addressed by Hassini and Vickson [5]. For order size $n=1$, they study the allocation problem in carousels that are grouped in a pod of two. They found several solutions that are close to optimal but could not determine the optimal allocation rule. In the last part of this paper, we discuss the allocation problem in a single-carousel system when the order size is greater than one. In particular, we show that the organ-pipe allocation is not optimal for large orders if a fixed-dwell point strategy is applied, that is, if after collecting an order, a carousel always returns to a fixed starting position. However, our simulation results indicate that the organ-pipe allocation might be optimal under the floating dwell point strategy, i.e., if after collecting an order, the carousel remains in its current position until a new order arrives.

The paper is organized as follows. In the next section, we formally describe the problem and provide the needed background. In Section 3, we derive the limiting distribution of the shortest travel time when the order size grows to infinity. In Section 4, we study analytic properties of the limiting distribution. The analysis of the number of items collected before a turn is presented in Section 5. The allocation issues are discussed in Section 6.

\section{Problem description}

We model a carousel as a circle of length 1 . The picker has a position at point zero, and he has to collect one order of $n$ items by moving along the circle at unit speed in either direction. The locations of the items are independent and identically distributed continuous random variables with probability density function $f(\cdot)$ which is positive and bounded on $[0,1]$. For $i=1, \ldots, n$, let $Y_{i}$ denote a location of the $i$ th item. Set $Y_{0}=0$, $Y_{n+1}=1$. Further, let $0=Y_{0: n}<Y_{1: n}<\cdots<Y_{n: n}<Y_{n+1: n}=1$ denote the order statistics of $Y_{0}, Y_{1}, \ldots, Y_{n+1}$. Then the picker's starting point and the positions of the $n$ items partition the circle into $n+1$ spacings

$$
D_{i, n}=Y_{i: n}-Y_{i-1: n}, \quad 1 \leq i \leq n+1 .
$$

We assume that the optimal picking strategy is adopted, that is, the picker chooses the shortest possible route. Now, let $T_{n}$ be the minimal travel time of the picker. Clearly, one can write $T_{n}$ in terms of the spacings $D_{1, n}, \ldots, D_{n+1, n}$. In fact, the formula remains the same as in [10] where we studied the case of uniformly distributed items:

$$
T_{n}=1-\max \left\{\max _{1 \leq j \leq n}\left\{D_{j, n}-Y_{j-1: n}\right\}, \max _{1 \leq j \leq n}\left\{D_{n+2-j, n}-\left(1-Y_{n+2-j: n}\right)\right\}\right\} .
$$

This formula has a clear interpretation. As was noticed by many authors (see e.g. [2]), the optimal route admits at most one turn. Further, for $j=1,2, \ldots, n$, the random variable 
$D_{j, n}-Y_{j-1: n}$ is the gain in travel time (compared to one full rotation) obtained by skipping the spacing $D_{j, n}$ and going back instead (ending in a clockwise direction). The same can be said about $D_{n+2-j, n}-\left(1-Y_{n+2-j: n}\right)$, but here the picker ends in a counterclockwise direction. Under the optimal strategy, the picker chooses the shortest route, i.e., largest possible gain.

It is convenient to write the travel time via spacings because various helpful properties of the spacings have been extensively studied in literature. The key reference is the classical paper of Pyke [13]. In our analysis, we shall exploit two useful results (1) and (3) quoted from this paper.

If the $Y_{i}$ 's have a uniform distribution, then the uniform spacings are distributed as normalized exponential random variables:

$$
\left(D_{1, n}, D_{2, n}, \ldots, D_{n+1, n}\right) \stackrel{d}{=}\left(X_{1} / S_{n+1}, X_{2} / S_{n+1}, \ldots, X_{n+1} / S_{n+1}\right) .
$$

Here $X_{1}, X_{2}, \ldots$ are independent exponentially distributed random variables with mean 1 and $S_{n+1}=X_{1}+\cdots+X_{n+1}$. In $[8,9,10]$ we analyzed various order picking strategies and, in particular, the optimal strategy, exploiting this property. Specifically, in order to derive the minimal travel time distribution, we first expressed the travel time in terms of exponential random variables and then applied the following lemma obtained in [6].

Lemma 2.1 Let $X_{1}, X_{2}, \ldots$ be i.i.d. exponential random variables with mean 1 . Define $S_{0}=0$ and $S_{j}=X_{1}+\cdots+X_{j}, j \geq 1$. Then for any $m=0,1, \ldots$,

$$
\max _{1 \leq j \leq m+1}\left\{X_{j}-S_{j-1}\right\} \stackrel{d}{=} \sum_{j=1}^{m+1}\left(2^{j}-1\right)^{-1} X_{j}
$$

In the current study, we deal with non-uniform spacings and thus (1) does not hold. However, when $n$ is large, one can apply a well-known asymptotic independence and exponentiality of non-uniform spacings. In Pyke [13], this result is written as follows. Let $F$ be a continuous distribution function of $Y_{i}, i=1, \ldots, n$, and $f$ be a corresponding density function. Denote $D_{n i}=(n+1) D_{i, n}, i=1, \ldots, n+1$. Then, if $i / n \rightarrow s$ and $j / n \rightarrow s$,

$$
\lim _{n \rightarrow \infty} F_{D_{n i}, D_{n j}}(x, y)=\left(1-e^{-f(s) x}\right)\left(1-e^{-f(s) y}\right) .
$$

This result can be extended to any finite set of spacings. In the next section, we use (3) to obtain a limiting behavior of $T_{n}$ when $n \rightarrow \infty$.

\section{The main result}

Let $X_{1}, X_{2}, \ldots, X_{1}^{\prime}, X_{2}^{\prime}, \ldots$ be independent exponential random variables with mean 1 . Then the following limiting result holds. 
Theorem 3.1 Let $f$ be a density function of $Y_{i}, i=1, \ldots, n$, and assume that $f$ is positive and bounded on $[0,1]$. Then

$$
(n+1)\left(1-T_{n}\right) \stackrel{d}{\longrightarrow} \max \left\{\frac{1}{f(0)} \sum_{j=1}^{\infty} \frac{1}{2^{j}-1} X_{j}, \frac{1}{f(1)} \sum_{j=1}^{\infty} \frac{1}{2^{j}-1} X_{j}^{\prime}\right\} \text { as } n \rightarrow \infty .
$$

The idea of the proof is as follows. First, we prove that the probability of making a turn after collecting $k$ items decreases exponentially with $k$. Thus, the travel time is determined essentially by a finite number of spacings close to the picker's starting position. For these spacings, we shall apply (3) and (2).

Proof of Theorem 3.1. First, note that the picker makes a turn after collecting the $(k-1)$ th item only in case of the event $\left[D_{k, n}-Y_{k-1: n}>0\right]$ or $\left[D_{n+2-k, n}>1-Y_{n+2-k: n}\right]$. The probability of the event $\left[D_{k, n}-Y_{k-1: n}>0\right]$ can be written as

$$
\mathbb{P}\left(D_{k, n}-Y_{k-1: n}>0\right)=\int_{0}^{1} \mathbb{P}\left(D_{k, n}>Y_{k-1: n} \mid Y_{k: n}=u\right) f_{Y_{k: n}}(u) d u .
$$

Further, for the conditional probability under the integral, we obtain

$$
\begin{aligned}
\mathbb{P}\left(D_{k, n}>\right. & \left.Y_{k-1: n} \mid Y_{k: n}=u\right)=\mathbb{P}\left(Y_{k-1: n} \leq u / 2 \mid Y_{k: n}=u\right) \\
& =\mathbb{P}\left(Y_{1}, \ldots, Y_{k-1} \leq u / 2 \mid Y_{1}, \ldots, Y_{k-1}<u\right)=\frac{[F(u / 2)]^{k-1}}{[F(u)]^{k-1}}
\end{aligned}
$$

Define $g_{1}(u)=F(u / 2) / F(u), u \in(0,1]$, and put $g_{1}(0)=\lim _{u \rightarrow 0} F(u / 2) / F(u)=1 / 2$. Note that $g_{1}(u)<1$ for all $u \in[0,1]$, since $F$ is a continuous distribution function and the density $f$ is strictly positive on $[0,1]$. Furthermore, function $g_{1}$ is defined on a compact set, and thus there exists $u_{1}^{*} \in[0,1]$ such that $\gamma_{1}=g_{1}\left(u_{1}^{*}\right)=\max _{0 \leq u \leq 1} g_{1}(u)$. Hence, $g_{1}\left(u_{1}\right) \leq \gamma_{1}<1$ for all $u_{1} \in[0,1]$. Thus, substituting (5) in (4), we obtain

$$
\mathbb{P}\left(D_{k, n}-Y_{k-1: n}>0\right)=\int_{0}^{1}\left(g_{1}(u)\right)^{k-1} f_{Y_{k: n}}(u) d u \leq \gamma_{1}^{k-1}
$$

Analogously, one can show that $\mathbb{P}\left(D_{n+2-k, n}>1-Y_{n+2-k: n}\right)$ is bounded by $\gamma_{2}^{k-1}$ where $\gamma_{2}<1$ is defined as $\max _{0 \leq u \leq 1} g_{2}(u)$ with $g_{2}(u)=(1-F((1-u) / 2)) /(1-F(1-u))$, $u \in[0,1)$, and $g_{2}(1)=\lim _{u \rightarrow 0} g_{2}(u)=1 / 2$.

We now approximate the minimal travel time by the travel time under the $m$-step strategy $[14,9]$. Exactly as under the optimal strategy, under the $m$-step strategy, the picker is allowed to turn only once. However, under the $m$-step strategy, the picker may turn after collecting at most $m$ items. Thus, the travel time $T_{n}^{(m)}$ under the $m$-step strategy can be written as

$$
T_{n}^{(m)}=1-\max \left\{\max _{1 \leq j \leq m+1}\left\{D_{j, n}-Y_{j-1: n}\right\}, \max _{1 \leq j \leq m+1}\left\{D_{n+2-j, n}-\left(1-Y_{n+2-j: n}\right)\right\}\right\} .
$$


For any fixed $m$, when $n$ goes to infinity, we use the result (3) extended for a finite set of spacings $\left\{D_{1, n}, \ldots, D_{m+1, n}, D_{n+1-m, n}, \ldots, D_{n+1, n}\right\}$. Then subsequently applying the continuous mapping theorem (see e.g. [4]) and Lemma 2.1, we obtain

$$
\begin{aligned}
(n+1)\left(1-T_{n}^{(m)}\right) \stackrel{d}{\rightarrow} \max \left\{\frac{1}{f(0)} \max _{1 \leq j \leq m+1}\left\{X_{j, n}-S_{j-1}\right\}, \frac{1}{f(1)} \max _{1 \leq j \leq m+1}\left\{X_{j, n}^{\prime}-S_{j-1}^{\prime}\right\}\right\}, \\
\stackrel{d}{=} \max \left\{\frac{1}{f(0)} \sum_{j=1}^{m+1} \frac{1}{2^{j}-1} X_{j}, \frac{1}{f(1)} \sum_{j=1}^{m+1} \frac{1}{2^{j}-1} X_{j}^{\prime}\right\} .
\end{aligned}
$$

Here $X_{1}, X_{2}, \ldots, X_{1}^{\prime}, X_{2}^{\prime}, \ldots$ are independent exponential random variables with mean 1 , $S_{0}=S_{0}^{\prime}=0, S_{i}=X_{1}+\cdots+X_{i}$, and $S_{i}^{\prime}=X_{1}^{\prime}+\cdots+X_{i}^{\prime}$, for all $i \geq 1$.

Now we can prove the limiting result. Denote

$$
J=\sum_{j=1}^{\infty} \frac{1}{2^{j}-1} X_{j}, \quad J^{\prime}=\sum_{j=1}^{\infty} \frac{1}{2^{j}-1} X_{j}^{\prime}, \quad J_{m}=\sum_{j=1}^{m+1} \frac{1}{2^{j}-1} X_{j}, \quad J_{m}^{\prime}=\sum_{j=1}^{m+1} \frac{1}{2^{j}-1} X_{j}^{\prime} .
$$

Further, for all $t \geq 0$, define

$$
P_{n}(t)=\mathbb{P}\left((n+1)\left(1-T_{n}\right)<t\right), \quad P_{n}^{(m)}(t)=\mathbb{P}\left((n+1)\left(1-T_{n}^{(m)}\right)<t\right),
$$

and

$$
P(t)=\mathbb{P}\left(\max \left\{\frac{1}{f(0)} J, \frac{1}{f(1)} J^{\prime}\right\}<t\right), \quad P^{(m)}(t)=\mathbb{P}\left(\max \left\{\frac{1}{f(0)} J_{m}, \frac{1}{f(1)} J_{m}^{\prime}\right\}<t\right) .
$$

Equation (8) can be now written as

$$
\lim _{n \rightarrow \infty} P_{n}^{(m)}(t)=P^{(m)}(t)
$$

To prove the statement of the theorem, note that $T_{n}$ can be smaller than $T_{n}^{(m)}$ only if it is optimal to turn after collecting $k>m$ items. The latter situation can occur only if either $\left[D_{k}>Y_{k-1, n}\right]$ or $\left[D_{n+2-k}>1-Y_{n+2-k, n}\right]$ holds for some $k>m$. Thus, for any $t \in[0,1]$, we have

$$
\begin{aligned}
0 & \leq P_{n}^{(m)}(t)-P_{n}(t)=\mathbb{P}\left(T_{n}^{(m)} \geq 1-t /(n+1), T_{n}<1-t /(n+1)\right) \leq \mathbb{P}\left(T_{n}<T_{n}^{(m)}\right) \\
& \leq \mathbb{P}\left(\left[\bigcup_{k=m+1}^{n}\left[D_{k}>Y_{k-1, n}\right]\right] \bigcup\left[\bigcup_{k=m+1}^{n}\left[D_{n+2-k}>1-Y_{n+2-k, n}\right]\right]\right) \\
& \leq \sum_{k=m+1}^{n} \mathbb{P}\left(D_{k}>Y_{k-1, n}\right)+\sum_{k=m+1}^{n} \mathbb{P}\left(D_{n+2-k}>1-Y_{n+2-k, n}\right) \leq \gamma_{1}^{m}+\gamma_{2}^{m} .
\end{aligned}
$$

Here the last inequality follows from (6). Now we can show that the $\operatorname{limit}_{\lim _{n \rightarrow \infty}} P_{n}(t)$ exists and equals $P(t)$. First, choose any $\varepsilon>0$ and fix $m$ large enough so that $\gamma_{1}^{m}+\gamma_{2}^{m}<$ 
$\varepsilon / 3$. It follows from (9) that it is possible to choose some $N>m$ such that for any $n_{1}, n_{2}>N$ holds $\left|P_{n_{1}}^{(m)}(t)-P_{n_{2}}^{(m)}(t)\right|<\varepsilon / 3$. Then, for such $n_{1}, n_{2}$, we get

$$
\begin{aligned}
\left|P_{n_{1}}(t)-P_{n_{2}}(t)\right| & \leq\left|P_{n_{1}}(t)-P_{n_{1}}^{(m)}(t)\right|+\left|P_{n_{1}}^{(m)}(t)-P_{n_{2}}^{(m)}(t)\right|+\left|P_{n_{2}}(t)-P_{n_{2}}^{(m)}(t)\right| \\
& \leq \gamma_{1}^{m}+\gamma_{2}^{m}+\varepsilon / 3+\gamma_{1}^{m}+\gamma_{2}^{m}<\varepsilon .
\end{aligned}
$$

Thus, according to the Cauchy criterion, $P_{n}(t)$ converges to a limit for any $t>0$. Then letting $n$ go to infinity in (10) and using (9) we obtain

$$
P^{(m)}(t) \leq \lim _{n \rightarrow \infty} P_{n}(t) \leq P^{(m)}(t)+\gamma_{1}^{m}+\gamma_{2}^{m}
$$

for an arbitrarily large $m$. Hence, for any $t>0$,

$$
\lim _{n \rightarrow \infty} P_{n}(t)=\lim _{m \rightarrow \infty} P^{(m)}(t)=P(t)
$$

Here the convergence in distribution also implies the convergence of moments. We shall compute the limiting expression for the moments in the next section.

\section{Analytic properties of the limiting distribution}

In the paper [10] we discuss in detail various interesting properties of the functional $J$ and its distribution function

$$
Q(t)=\mathbb{P}(J<t), \quad t \geq 0 .
$$

The statements of the next theorem are quoted from [10].

Theorem 4.1 (from [10])

(i) The distribution function $Q$ is given by

$$
Q(t)=1-\sum_{j=1}^{\infty}(-1)^{j-1} 2^{j} \exp \left\{-\left(2^{j}-1\right) t\right\} \prod_{l=1}^{j} \frac{1}{2^{l}-1}
$$

(ii) $Q$ is infinitely often differentiable and all of its derivatives $Q^{(k)}$ vanish at the origin.

(iii) The asymptotic behavior of $Q$ in a small positive neighborhood of zero is given by

$$
Q(t) \sim \frac{\sqrt{\log 2} \prod_{j=1}^{\infty}\left(1-2^{-j}\right)^{2}}{2^{1 / 8} 2 \pi \tilde{\vartheta}_{3}(\operatorname{frac}\{\psi(t)\})} \exp \left\{-\frac{\log 2}{2}[\psi(t)]^{2}\right\} \cdot t^{-\left(\frac{1}{2}+\frac{1}{\log 2}\right)}, \quad \text { as } t \rightarrow+0,
$$


where $\psi(t)=(\log 2)^{-1}[\log (1 / t)+\log \log (1 / t)-\log \log 2]$ and

$$
\begin{aligned}
\tilde{\vartheta}_{3}(\theta) & =1+2 \sum_{k=1}^{\infty} \exp \left\{-2 k^{2} \pi^{2} / \log 2\right\} \cos \{2 k \pi(1 / 2-\theta)\} \\
& =\vartheta_{3}\left(\pi(1 / 2-\theta), \exp \left\{-2 \pi^{2} / \log 2\right\}\right) .
\end{aligned}
$$

Here $\vartheta_{3}$ is a theta function

$$
\vartheta_{3}(z, q)=1+2 \sum_{k=1}^{\infty} q^{k^{2}} \cos (2 k z)
$$

Statements (ii) and (iii) reveal most remarkable properties of the function $Q$. Clearly, this function is not analytic at the origin and has a peculiar asymptotic behavior that involves oscillations described by the Jacobi's theta-function. It is easy to establish the relation between the distribution functions $P$ and $Q$. It follows from the definition of $P$ that

$$
P(t)=\mathbb{P}\left(\frac{1}{f(0)} J<t, \frac{1}{f(1)} J^{\prime}<t\right)=Q(f(0) t) Q(f(1) t), \quad t \geq 0 .
$$

Equation (11) together with Theorem 4.1 provides the explicit expression for $P$ as well as its properties and asymptotic behavior in a positive neighborhood of zero.

Using (11) and Theorem 4.1(i) one can also determine the limiting expression for the moments. For the $k$-th moment we find

$$
\begin{aligned}
& \lim _{n \rightarrow \infty} \mathbb{E}\left[(n+1)\left(1-T_{n}\right)\right]^{k}=k ! \sum_{\alpha=0,1} f(\alpha) \sum_{j=1}^{\infty}(-1)^{j-1} \frac{2^{j}}{\left(2^{j}-1\right)^{k}} \prod_{l=1}^{j} \frac{1}{2^{l}-1} \\
& -k ! \sum_{\substack{\alpha, \beta=0,1 \\
\alpha \neq \beta}} f(\alpha) \sum_{j=1}^{\infty} \sum_{i=1}^{\infty}(-1)^{i+j} \frac{2^{i+j}}{\left[f(\alpha)\left(2^{i}-1\right)+f(\beta)\left(2^{j}-1\right)\right]^{k+1}} \prod_{l=1}^{j} \frac{1}{2^{l}-1} \prod_{r=1}^{i-1} \frac{1}{2^{r}-1} .
\end{aligned}
$$

An equivalent expression for the expectation can be obtained as

$$
\begin{aligned}
& \mu=\lim _{n \rightarrow \infty} \mathbb{E}\left[(n+1)\left(1-T_{n}\right)\right]=\int_{0}^{\infty}[1-Q(f(0) t) Q(f(1) t)] d t= \\
& \sum_{\alpha=0,1} \frac{1}{f(\alpha)} \sum_{j=1}^{\infty} \frac{1}{2^{j}-1}-\sum_{i=1}^{\infty} \sum_{j=1}^{\infty}(-1)^{i+j} \frac{2^{i+j}}{\left(2^{i}-1\right) f(0)+\left(2^{j}-1\right) f(1)} \prod_{l=1}^{j} \frac{1}{2^{l}-1} \prod_{r=1}^{i} \frac{1}{2^{r}-1} .
\end{aligned}
$$

For given $f(0), f(1)$, the value of $\mu$ can be easily computed. Then the average minimal travel time can be approximated as

$$
\mathbb{E}\left(T_{n}\right) \approx 1-\frac{\mu}{n+1} .
$$




\section{Number of steps before a turn}

As we discussed earlier, the optimal route implies at most one turn. In [9] we proved that if the items' locations are independent and uniformly distributed then the number of items collected before a turn converges to a geometrically distributed random variable with parameter $1 / 2$. In this section, we will show that this surprising result also holds for non-uniform items' locations. Moreover, the number of items collected before a turn and the travel time turn out to be asymptotically independent as the order size $n$ goes to infinity.

Denote by $K_{n}$ and $K_{n}^{(m)}$ the number of items collected before a turn when collecting an order of $n$ items under the optimal strategy and the $m$-step strategy, respectively. If there is no turn, this numbers are set equal to zero. Observe that the number $K_{n}^{(m)}+1$ equals to one of the values $j_{1}$ or $j_{2}$ where either the first or the second internal maximum in (7) is achieved. The choice of $j_{1}$ or $j_{2}$ depends on whether the first or the second internal maximum is larger, or, in other words, whether the picker collects an order finishing clockwise or counterclockwise. Denote by $C$ an event that the picker's last movement is directed clockwise. Then $K_{n}^{(m)}$ can be formally written as

$$
\arg \max _{1 \leq j \leq m+1}\left\{D_{j, n}-Y_{j-1: n}\right\} \mathbf{1}_{\{C\}}+\arg \max _{1 \leq j \leq m+1}\left\{D_{n+2-j, n}-\left(1-Y_{n+2-j: n}\right)\right\} \mathbf{1}_{\{\bar{C}\}}-1,
$$

or, equivalently, as

$$
\begin{aligned}
& \arg \max _{1 \leq j \leq m+1}\left\{(n+1) D_{j, n}-(n+1) Y_{j-1: n}\right\} \mathbf{1}_{\{C\}} \\
& \quad+\arg \max _{1 \leq j \leq m+1}\left\{(n+1) D_{n+2-j, n}-(n+1)\left(1-Y_{n+2-j: n}\right)\right\} \mathbf{1}_{\{\bar{C}\}}-1 .
\end{aligned}
$$

Here $\mathbf{1}_{\{\cdot\}}$ is the indicator function. In [9] we proved that if locations of the items are uniformly distributed then $K_{n}^{(m)}$ is independent of $T_{n}^{(m)}$ for all $n>2 m$, and we derived the distribution of $K_{n}^{(m)}$. These results are based on Corollary 3.2 from [9] that provides the formula

$$
\mathbb{P}\left(\arg \max _{1 \leq j \leq m+1}\left\{X_{j}-S_{j-1}\right\}-1=k\right)=\frac{1}{2^{k+1}-2^{k-m}},
$$

and establishes the independence of

$$
\arg \max _{1 \leq j \leq m+1}\left\{X_{j}-S_{j-1}\right\}
$$

and

$$
\max _{1 \leq j \leq m+1}\left\{X_{j}-S_{j-1}\right\} .
$$

The next lemma states that similar results hold asymptotically for independent items locations with any continuous distribution on $[0,1]$.

Lemma 5.1 For any $m \geq 0$, it holds that (i) $(n+1)\left(1-T_{n}^{(m)}\right)$ and $K_{n}^{(m)}$ are asymptotically independent as $n \rightarrow \infty$; 
(ii) if $n \rightarrow \infty$, then $K_{n}^{(m)} \stackrel{d}{\rightarrow} K^{(m)}$ where

$$
\mathbb{P}\left(K^{(m)}=k\right)=\frac{1}{2^{k+1}-2^{k-m}}=\frac{1}{2^{k+1}}+\frac{1}{2^{m+1}\left(2^{k+1}-2^{k-m}\right)}, \quad k=0, \ldots, m .
$$

Proof. We first let $n \rightarrow \infty$ in (12) and apply (3) together with the continuous mapping theorem. Then we see that $K_{n}^{(m)}$ converges in distribution to $K^{(m)}$ given by

$$
\arg \max _{1 \leq j \leq m+1}\left\{\frac{1}{f(0)}\left[X_{j}-S_{j-1}\right]\right\} \mathbf{1}_{\{C\}}+\arg \max _{1 \leq j \leq m+1}\left\{\frac{1}{f(1)}\left[X_{j}^{\prime}-S_{j-1}^{\prime}\right]\right\} \mathbf{1}_{\{\bar{C}\}}-1,
$$

where $C$ reduces to the event

$$
\left[\frac{1}{f(0)} \max _{1 \leq j \leq m+1}\left\{X_{j}-S_{j-1}\right\} \geq \frac{1}{f(1)} \max _{1 \leq j \leq m+1}\left\{X_{j}^{\prime}-S_{j-1}^{\prime}\right\}\right] .
$$

Note that the independence of (15) and (14) implies that the distribution of $K^{(m)}$ does not depend on the maxima involved in the event $C$. Thus, for $0 \leq k \leq m$, we subsequently use the total probability formula, the independence of (15) and (14) and the formula (13) to obtain

$$
\begin{aligned}
& \mathbb{P}\left(K^{(m)}=k\right)=\mathbb{P}(C) \mathbb{P}\left(\arg \max _{1 \leq j \leq m+1}\left\{X_{j}-S_{j-1}\right\}=k-1 \mid C\right) \\
& \quad+\mathbb{P}(\bar{C}) \mathbb{P}\left(\arg \max _{1 \leq j \leq m+1}\left\{X_{j}^{\prime}-S_{j-1}^{\prime}\right\}=k-1 \mid \bar{C}\right) \\
& \quad=\mathbb{P}\left(\arg \max _{1 \leq j \leq m+1}\left\{X_{j}-S_{j-1}\right\}=k-1\right)=\frac{1}{2^{k+1}-2^{k-m}} .
\end{aligned}
$$

Note that the derivation implies the asymptotic independence of $K_{n}^{(m)}$ and $(n+1)\left(1-T_{n}^{(m)}\right)$, and thus we have proved both statements of the lemma.

We are now ready to establish asymptotic properties of the number of items collected before the turn under the optimal strategy.

Theorem 5.2 (i) For any fixed $k \geq 0$,

$$
\lim _{n \rightarrow \infty} \mathbb{P}\left(K_{n}=k\right)=2^{-(k+1)}, \quad k=0,1, \ldots
$$

(ii) $(n+1)\left(1-T_{n}\right)$ and $K_{n}$ are asymptotically independent when $n$ goes to infinity.

Proof. In order to prove (i), observe that for $0 \leq k \leq m$,

$$
\mathbb{P}\left(K_{n}^{(m)}=k\right)-\mathbb{P}\left(K_{n}>m\right) \leq \mathbb{P}\left(K_{n}=k\right) \leq \mathbb{P}\left(K_{n}^{(m)}=k\right) .
$$

Using (10) we obtain

$$
\mathbb{P}\left(K_{n}>m\right)=\mathbb{P}\left(T_{n}<T_{n}^{(m)}\right) \leq \gamma_{1}^{m}+\gamma_{2}^{m}
$$


Further, it follows from Lemma 5.1 that $K_{n}^{(m)} \stackrel{d}{\rightarrow} K^{(m)}$ as $n \rightarrow \infty$. Now, for any fixed $k \geq 0$, we can apply the Cauchy criterion for $\mathbb{P}\left(K_{n}=k\right)$ exactly as it was done for $P_{n}(t)$ in the last part of the proof of Theorem 3.1, herewith showing that $\lim _{n \rightarrow \infty} \mathbb{P}\left(K_{n}=k\right)$ exists and equals $\lim _{m \rightarrow \infty} \mathbb{P}\left(K^{(m)}=k\right)=2^{-(k+1)}$ where the last equality follows directly from Lemma 5.1 (ii).

The proof of (ii) is along the same lines. We first write

$$
\begin{array}{r}
0 \leq \mathbb{P}\left(K_{n}^{(m)}=k, T_{n}^{(m)} \geq 1-t /(n+1)\right)-\mathbb{P}\left(K_{n}=k, T_{n} \geq 1-t /(n+1)\right) \\
\leq \mathbb{P}\left(T_{n}^{(m)}>T_{n}\right) \leq \gamma_{1}^{m}+\gamma_{2}^{m} .
\end{array}
$$

Using again the Cauchy criterion and the asymptotic independence of $K_{n}^{(m)}$ and $(n+1)(1-$ $\left.T_{n}^{(m)}\right)$ as $n \rightarrow \infty$, one can show that $\lim _{n \rightarrow \infty} \mathbb{P}\left(K_{n}=k, T_{n}<1-t /(n+1)\right)$ exists and equals

$$
\begin{aligned}
& \lim _{m \rightarrow \infty} \lim _{n \rightarrow \infty} \mathbb{P}\left(K_{n}^{(m)}=k, T_{n}^{(m)}<1-t /(n+1)\right) \\
& =\lim _{m \rightarrow \infty} \lim _{n \rightarrow \infty} \mathbb{P}\left(K_{n}^{(m)}=k\right) \lim _{m \rightarrow \infty} \lim _{n \rightarrow \infty} \mathbb{P}\left(T_{n}^{(m)}<1-t /(n+1)\right)=2^{-(k+1)} P(t) .
\end{aligned}
$$

The asymptotic independence of $K_{n}$ and $(n+1)\left(1-T_{n}\right)$ now readily follows from (i) and Theorem 3.1.

\section{Optimal allocation rules}

This section addresses an interesting open problem of optimal allocation of items in a carousel. We consider two cases: fixed dwell point strategy and floating dwell point strategy. Under the fixed dwell point strategy, the carousel returns to its starting position every time after collecting an order. On the contrary, under the floating dwell point strategy, the end point of picking an order becomes a starting point for collecting the next order.

In paper [3] it was shown that if an order consists of one item then the following allocation policy is optimal for both fixed and floating dwell point strategies:

Organ-pipe (OP) allocation policy. Place the most frequently asked item at the bin located at the starting point of the picker. Repetitively place next most frequently asked item alternating between the positions to the left and to the right of the already placed item.

For orders consisting of multiple items, the problem of optimal allocation has not been resolved. In this section, we shall use our asymptotic results on the travel time in order to provide new insights into the allocation problem.

\subsection{Fixed dwell point strategy}

Consider a carousel system with fixed dwell point strategy. Under this strategy, the carousel returns to its starting position every time after collecting an order. Such strategy 
may be used if a picker needs some time for sorting/labeling the order while the carousel rotates to its starting point. Besides, fixed dwell point strategy might be useful if one picker operates several carousels utilized in a pod $[15,16,12,11]$. Under the fixed dwell point strategy, an optimal picking of one order is equivalent to the optimal picking of several orders in a row. Therefore, one can apply the results on the minimal travel time derived in previous sections.

As mentioned above, the OP allocation is clearly optimal if an order consists of one item. However, in practice, it is usually not the case. Consider an order consisting of multiple items. Then it follows immediately from Theorem 3.1 that for large enough $n$, the random variable $T_{n}$ stochastically increases with $f(0)$ and $f(1)$. Hence, if orders are large, then less frequently asked items have to be stored close to the picker's starting point. This conclusion has an easy intuitive explanation illustrated in Figure 1, where we depict
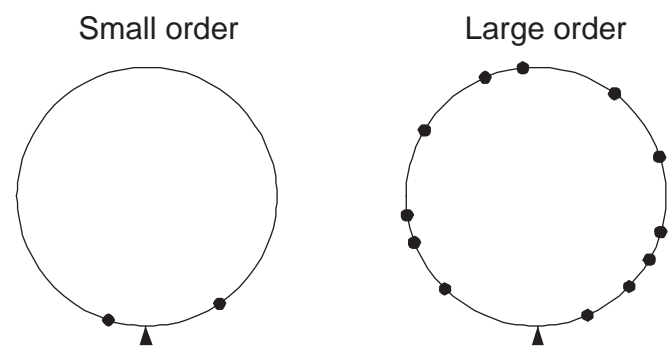

Figure 1: Distribution of the items on a carousel in case of small and large orders

a typical layout for small and large orders. If an order is small, then it is reasonable to store most frequently asked items close by, hoping that the whole order can be collected by traveling only a small part of the circle. However, if an order is large, then, most probably, the picker has to cover the major part of the circle anyway. Hence, in this case, the travel time can be reduced only by skipping a large spacing close to the picker's starting point. Such large spacing is more likely to occur if the items that are stored close to the picker's starting point, are not been demanded frequently. This suggests the next two allocation strategies that may suite for collecting large orders under the fixed dwell point strategy.

Reversed organ-pipe (ROP) allocation policy. Place the least frequently asked item at the bin located at the starting point of the picker. Repetitively place next least frequently asked item alternating between the positions to the left and to the right of the already placed item.

Monotone allocation policy. Place the least frequently asked item at the bin located at the starting point of the picker. Repetitively place next least frequently asked item to the right of the already placed item.

We shall also consider a random allocation policy that models, for instance, the allocation in the alphabetic order. 
Random allocation policy. Repetitively place an item in a randomly chosen available bin.

In Figure 2, we illustrate the simulation results for the mean travel time in a carousel with 100 pick faces (shelves or drawers). The frequency $f_{i}$ with which an item $i=1, \ldots, 100$

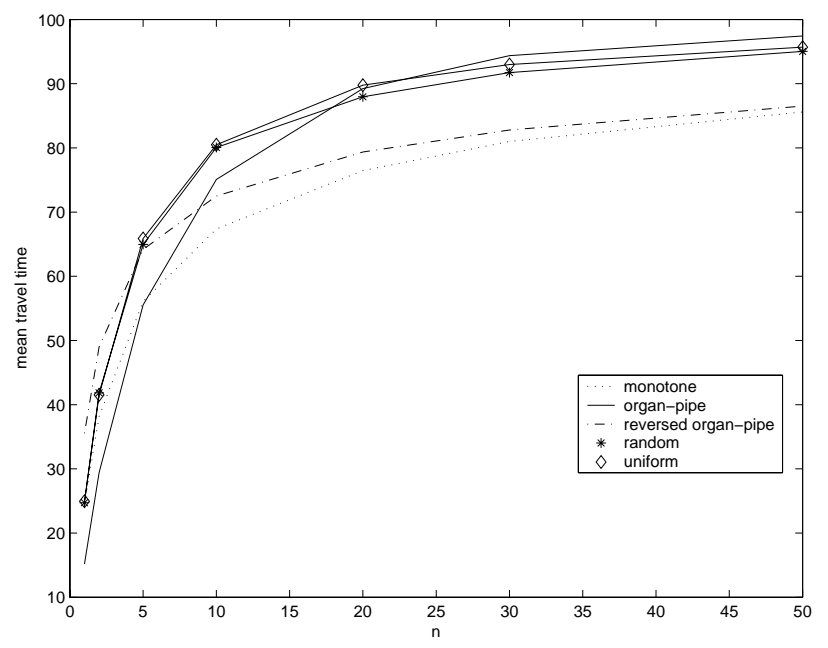

Figure 2: Average mean travel time under the fixed dwell point strategy in a carousel with 100 pick faces

is demanded is modeled according to the truncated normal distribution

$$
f_{i}=\frac{\int_{i-1}^{i} e^{-\frac{(x-\mu)^{2}}{2 \sigma^{2}}} d x}{\int_{0}^{100} e^{-\frac{(x-\mu)^{2}}{2 \sigma^{2}}} d x}
$$

where we chose $\mu=50$ and $\sigma=20$. One can see that the OP policy is only suitable for very small orders. Already for $n=5$, the monotone policy outperforms the OP policy, and remains optimal also for large orders.

One may wonder why the ROP policy performs worse than the monotone allocation. Intuitively, it should be the case because the monotone allocation maximizes the chance of having a large spacing next to the picker's starting point. Note also that the OP policy is the most unstable one. For small orders, and especially for the orders consisting of one item only, this policy is clearly the best. However, for large orders, it performs even worse than the random allocation.

Certainly, the results would change if the frequencies are chosen differently. For instance, in Figure 2 we depict the average travel time when locations of the items are uniformly distributed. Note that for uniformly distributed items the allocation problem is not relevant. We see however that the average travel time for uniformly distributed items is larger than for non-uniformly distributed items with a reasonable allocation policy. This 
suggests that in a carousel pod, it is not reasonable to accumulate items of similar demand frequencies on the same carousel unit. One should rather make sure that each carousel accommodates items of diverse demand frequencies. Our results indicate that such policy may considerably improve the throughput of the picker.

\subsection{Floating dwell point strategy}

The floating dwell point strategy implies that the picker starts collecting an order from the same point where he finished collecting the previous one. Such strategy prevents unnecessary rotation of the carousel. Mathematically, the analysis of the floating dwell point strategy is more difficult because the sequence of the picker's starting positions now constitutes a Markov process, and the travel times needed to collect subsequent orders become dependent.

For $i \geq 0$, denote by $V_{i}$ the position of the picker after collecting the $i$ th order. In a model with a finite number $N$ of carousel faces, the Markov chain $\left\{V_{i}\right\}_{i \geq 0}$ has a finite state space, so the stationary distribution $V$ obviously exists. If a carousel is modeled as a circle of length 1 , then the state space becomes $[0,1)$, and the existence of a stationary distribution can be proved by using a notion of Harris recurrence. Indeed, let $\phi^{(k)}(y \mid x)$ be the conditional probability density function of $V_{i+k}$ conditioned on the event $\left[V_{i}=x\right]$, where $i \geq 0$. Observe that $\phi^{(1)}(y \mid x)=0$ may equal zero, for instance when $x=y$. However, if $f$ is positive and bounded on $[0,1]$ then for all $k>1 ; x, y \in[0,1)$, there exist $\varepsilon_{k}>0$ and $C_{k}<\infty$ such that $\varepsilon_{k}<\phi^{(k)}(y \mid x)<C_{k}$. Now consider a set $R=[0,1)$. Since this set equals to the state space itself, $R$ is obviously recurrent. Further, for any $x \in R, B \subset R$, and $i \geq 0$, we write

$$
\begin{aligned}
P^{(2)}(x, B) & =\mathbb{P}\left(V_{i+2} \in B \mid V_{i}=x\right)=\int_{B} \phi^{(2)}(y \mid x) d y \\
& =\int_{B} \frac{\phi^{(2)}(y \mid x)}{\phi^{(2)}(y \mid 0)} \phi^{(2)}(y \mid 0) d y \geq \frac{\varepsilon_{2}}{C_{2}} \int_{B} \phi^{(2)}(y \mid 0), d y .
\end{aligned}
$$

Thus, $R$ is a regeneration set according to the definition in [1, p.198] with $r=2, \varepsilon=\varepsilon_{2} / C_{2}$, and $\lambda(B)=P^{(2)}(0, B)$. Hence, $\left\{V_{i}\right\}_{i \geq 0}$ is a Harris chain, and thus there exists a unique stationary distribution $V$.

Unfortunately, the stationary distribution of $\left\{V_{i}\right\}_{i \geq 0}$ is hard to derive for both discrete and continuous models. Below in Figure 3 we depict some simulation results for different order sizes in case of the OP allocation policy on a carousel with 100 storage faces. For orders of size 1, the distribution of $V$ is clearly the same as the distribution of items' locations. However, for $n>1$, we clearly observe that $V$ has a two-modal distribution, and this property exacerbates with growth of $n$. Thus, with the OP allocation policy, the picker will almost never reside at the most frequently demanded drawer, and there would be two symmetric moderately demanded set of drawers where the picker would stop most frequently.

In Figure 4 we compare an average travel time needed to collect an order under the floating dwell point strategy for different allocation policies. We did not consider a ROP 


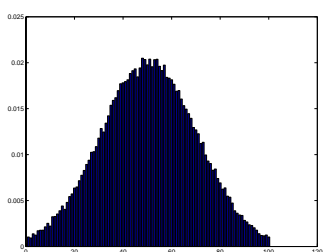

$n=1$

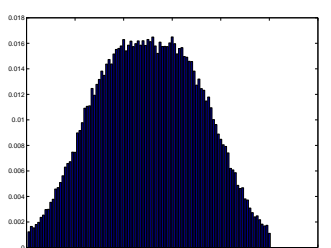

$n=2$

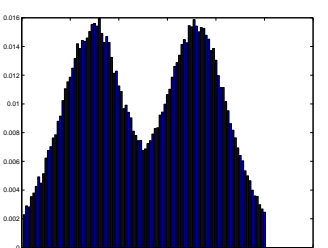

$n=5$

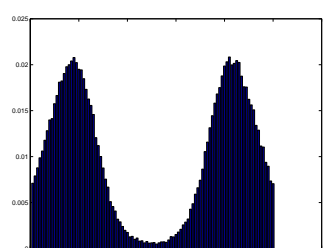

$n=20$

Figure 3: Probability density function of the picker's position in a stationary regime

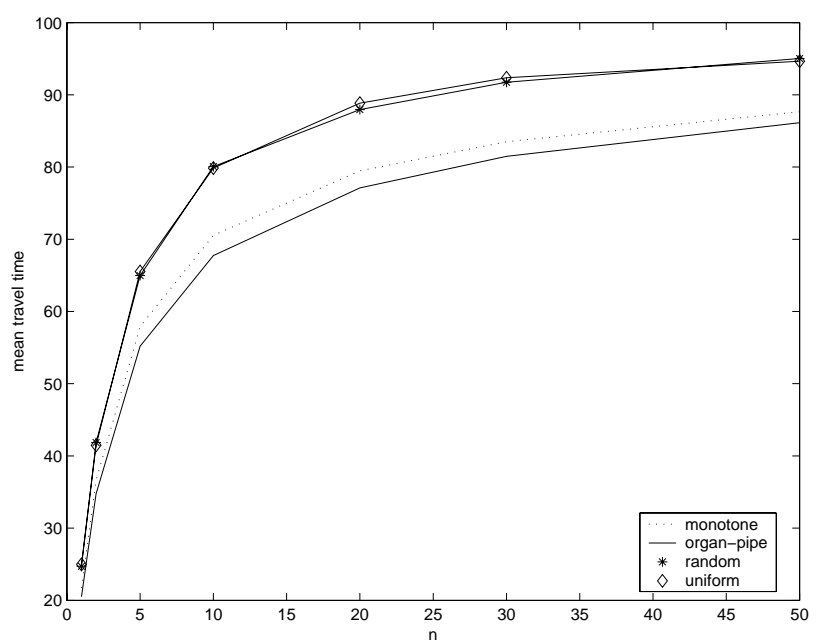

Figure 4: Average mean travel time under the floating dwell point strategy in a carousel with 100 pick faces

policy here because for the floating dwell point strategy in a stationary regime there is no difference between the ROP and the OP policy. We see that the OP policy now is clearly better that any other policy. Thus one may guess that this policy could be optimal for orders of any size. It is also apparent that the average travel time for the random allocation policy is almost the same as for uniformly distributed item's locations. Since this travel time is larger than for the OP allocation, we can again conclude that assigning items with diverse demand frequencies on the same carousel results in reduced travel times. This is in lines with results of Hassini and Vickson [5] on optimal storing of products in carousels grouped in pods of two. For one-item orders, their nearly-optimal solutions are characterized by variability of demand frequencies in each of the two carousels.

\section{References}

[1] S. Asmussen (2003) Applied Probability and Queues. Second edition. Springer-Verlag, New York. 
[2] Bartholdi, J.J.III and Platzman, L.K. (1986) Retrieval strategies for a carousel conveyor. IIE Transactions 18, 166-173.

[3] J. Bengu (1995) An optimal storage assignment for automating rotating carousels. IIE Transactions27, 105-107.

[4] P. Billingsley (1999) Convergence of Probability Measures. Second edition. Wiley, New York.

[5] E. Hassini and R. Vickson (2003) A two-carousel storage location problem. Comput. Oper. Res. 30(4), 527-539

[6] N. Litvak (2001) Some peculiarities of exponential random variables. J. Appl. Probab. 38, 787-792.

[7] N. Litvak, I.J.B.F. Adan, J. Wessels And W.H.M. Zijm. (2001) Order picking in carousel systems under the nearest item heuristic. Probab. Engrg. Inform. Sci. 15(2), 135-164.

[8] N. Litvak AND I. AdAN (2001) The travel time in carousel systems under the nearest item heuristic. J. Appl. Prob. 38(1), 45-54.

[9] N. Litvak AND I. AdAn (2002) On a class of order pick strategies in paternosters. Oper. Res. Lett. 30, 377-386.

[10] N. Litvak AND W.R. VAn Zwet (2004) On the minimal travel time needed to collect $n$ items on a circle. Ann. Appl. Probab. 14, 881-902.

[11] R.D. Meller AND J.F. Klote (2004) A throughput model for carousel/VLM pods. IIE Transactions 36(8), 725 - 741.

[12] B.C. Park, J.Y. Park and R.D. Foley (2003) Carousel system performance. J. Appl. Probab.40(3), 602-612.

[13] Pyke, R. (1965) Spacings. J. Roy. Statist. Soc. Ser. B 27, 395-449.

[14] Rouwenhorst, B., van Den Berg, J.P., van Houtum, G.J. And Zism, W.H.M. (1996) Performance analysis of a carousel system. In Progress in Material Handling Research: 1996, The Material Handling Industry of America, Charlotte, NC, 495-511.

[15] M. Vlasiou, I. Adan and J. Wessels A Lindley-type equation arising from a carousel problem. EURANDOM Report 2004-026.

[16] M. Vlasiou AND I. AdAn An alternating service problem. EURANDOM Report 2004-033.

[17] Y.-W. Wan and R.W. Wolff (2004) Picking clumpy orders on a carousel. Probab. Engrg. Inform. Sci.18(1), 1-11. 